\title{
MODELOS DESCRIPTIVOS DE DISTRIBUCIÓN DE MADERA JUVENIL Y MADURA EN Robinia pseudoacacia
}

\section{DESCRIPTIVE MODELS OF DISTRIBUTION OF JUVENILE AND MATURE WOOD IN Robinia pseudoacacia}

\author{
Ana Clara Cobas ${ }^{1}$, Silvia Monteoliva ${ }^{2,,}$
}

\author{
RESUMEN
}

Los objetivos del trabajo fueron 1- Determinar los patrones de variación axial y radial de la densidad de la madera y longitud de fibras en el fuste de Robinia pseudoacacia, 2- Determinar la edad de transición entre madera juvenil y madera madura, 3-Construir un modelo descriptivo de distribución de la madera juvenil en el fuste en base a la densidad de madera y longitud de fibras. Se cortaron rodajas a 4 alturas en el fuste $(0,3 \mathrm{~m} ; 1,3 \mathrm{~m} ; 2,3 \mathrm{~m}$ y $4 \mathrm{~m}$ a partir del suelo) en 10 árboles (7-15 años) implantados en Buenos Aires, Argentina. Se determinó la densidad normal ${ }_{12 \%}$ y la longitud de fibras en probetas radiales obtenidas cada 3 anillos de crecimiento en todas las alturas. La densidad de la madera mostró una homogeneidad de los valores a lo largo del fuste comercial (variación axial), con un rango que la clasifica como madera semi-pesada $\left(750-823 \mathrm{~kg} / \mathrm{m}^{3}\right)$. La longitud de fibras mostró aumento radial de sus valores de médula a corteza y sin variaciones axiales. El modelo de madera juvenil se asemeja al modelo de cilindro central pero de menor diámetro en el ápice. El cono juvenil se formaría hasta la edad de 3-6 años.

Palabras clave: Acacia blanca, densidad de la madera, longitud de fibras, patrones de variación, propiedades físicas.

\begin{abstract}
The objectives of the work were 1- to determine the patterns of axial and radial variation of wood density and fiber length in the loge of Robinia pseudoacacia, 2- to determine the transition age between juvenile and mature wood, 3-Construct a descriptive model of distribution of juvenile wood in the $\log$ based on wood density and fiber length. Slices were cut at 4 heights in the trunk $(0,3 \mathrm{~m} ; 1,3 \mathrm{~m}$; $2,3 \mathrm{~m}$ y $4 \mathrm{~m}$ above the ground) in 10 trees from Buenos Aires-Argentina (7-15 years old) implanted in the Province of Buenos Aires. The normal density ${ }_{12 \%}$ and fibre length were determined in radial specimens obtained every 3 growth rings at all heights. The density showed homogeneity values along the commercial shaft, with a range of magnitude that classified as semi-heavy wood $\left(750-823 \mathrm{~kg} / \mathrm{m}^{3}\right)$. The fiber length showed radial increase of their values from pith to bark and no axial variations. The model of juvenile wood resembles the model of central cylinder but of smaller diameter at the apex. The juvenile cone would form until the age of 3-6 years.
\end{abstract}

Keywords: Black locust, fiber length, patterns of variations, physical properties, wood density.

1Centro de Investigaciones y Transferencia del Noroeste de la provincia de Buenos Aires (CITNOBA) - Consejo Nacional de Investigaciones Científicas y Técnicas (CONICET), Junín, Argentina.

2Facultad de Ciencias Agrarias y Forestales, Universidad Nacional de La Plata, CONICET, La Plata, Argentina.

•Corresponding autor: smonteoliva@yahoo.com.ar

Received: 20.04.2017 Accepted: 30.12.2017 


\section{INTRODUCCIÓN}

Los valores de las propiedades de la madera están influenciados por la posición dentro del árbol. Se han demostrado patrones de variación radial (médula- corteza) y patrones de variación axial (baseápice) en diferentes especies, asociados a distintas edades fisiológicas del cambium y por lo tanto a la formación de madera juvenil y madura (Matyas and Peszlen 1997, Zobel and Sprague 1998, De Bell et al. 2002). El leño juvenil fue definido para las coníferas como la zona más cercana al centro del árbol que se extiende desde la base hasta el ápice. El tamaño de este cilindro central puede variar considerablemente entre árboles de una misma especie y edad (Zobel and Jett 1995) y también dependiendo de la propiedad que se utilice para su delimitación (Tasissa and Burkhart 1998, Cobas et al. 2013a, Cobas et al. 2014). La literatura indica que la madera juvenil está asociada a una edad fisiológica temprana de las células cambiales, presenta menor densidad y elementos fibrosos más cortos que la zona de madera madura (Zobel and Sprague 1998, De Bell et al. 2002).

Para Latifoliadas se han reportado trabajos de revisión de modelos de variación para algunos géneros en los que se involucran especies tanto de porosidad circular como difusa (Quercus, Eucalyptus, Populus, Salix, Tectona, Gmelina, Alnus, Tilia), en los cuales se pone de manifiesto la complejidad del tejido de las angiospermas y la gran diversidad de especies que comprende (Panshin and De Zeeuw 1980, Fukasawa 1984, Downes et al. 1997, Gartner et al. 1997, Bhat et al. 2001, De Bell et al. 2002, Barnett and Jeronimidis 2003, Moya et al. 2009, Moya and Tomazello Fo 2009, Downes et al. 2009, Tsuchiya and Furukawa 2009, Kojima et al. 2009a, Cobas et al. 2013a, Cobas et al. 2014). Para Robinia pseudoacacia (acacia blanca) existen muy pocos antecedentes de estudios de variación de sus propiedades a lo largo del fuste (Stringer and Olson 1987, Adamapoulos et al. 2007, Adamapoulos et al. 2010) y de la determinación de la edad de transición entre madera juvenil y madura (Adamopoulos and Voulgaridis 2002). No hay datos de patrones de variación publicados para acacia blanca implantada en Argentina ni de la determinación de la edad de transición entre los dos leños.

Estas variaciones de las propiedades de la madera tienen un efecto importante sobre las características y el rendimiento de las pulpas y los productos del papel, así como también en la resistencia y la utilidad de sus productos sólidos (Downes et al. 1997). Adamopoulos et al. (2007) publicaron que las propiedades de la madera juvenil de acacia blanca cultivada en Grecia presentó una menor resistencia a la flexión (MOR y MOE) y dureza, que la madera madura ante similares valores de densidad. Para otras especies comerciales, Cobas et al. (2013b) reportaron que las pulpas quimimecánicas de Salicáceas con mayor porcentaje de madera juvenil (100 \%) presentaron los valores más elevados en las propiedades mecánicas de las pulpas (tracción y rasgado fundamentalmente), con un comportamiento inverso en el caso de las propiedades ópticas. Se genera entonces un compromiso en la optimización de la edad de corte de las plantaciones con determinados usos, para responder así a las diferentes necesidades y requerimientos del producto industrial a producir.

En Argentina, el mercado de la madera de acacia blanca es pequeño, si bien existe un uso tradicional de la misma en carpintería de obra y construcciones rurales (Keil et al. 2011). El conocimiento de los patrones de variación axial y radial de las propiedades de las maderas y la zona de demarcación entre la madera juvenil y madura permitirá delimitar el turno de corta de acuerdo a la propiedad que se quiera potenciar y el destino final del producto a obtener (Larson et al. 2001, Kojima et al. 2009b).

Los objetivos del trabajo fueron 1- Determinar los patrones de variación axial y radial de la densidad de la madera y longitud de fibras en el fuste de Robinia pseudoacacia, 2- Determinar la edad de transición entre madera juvenil y madera madura, 3-Construir un modelo descriptivo de distribución de la madera juvenil en el fuste en base a la densidad de madera y longitud de fibras.

\section{MATERIALES Y MÉTODOS}

Se seleccionaron 10 árboles de Robinia pseudoacacia L. con edades comprendidas entre 7-15 años en un monte de reparo, sin manejo silvícola, ubicado en el establecimiento Lanfranchi, Chivilcoy, Pcia 


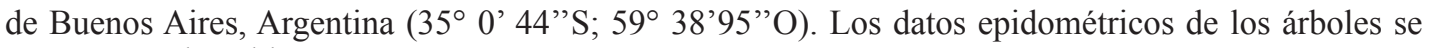
muestran en la Tabla 1.

Tabla 1. Altura total (AT), altura comercial hasta diámetro mínimo de $5 \mathrm{~cm}(\mathrm{AC})$ y diámetro a la altura de pecho (DAP) de los árboles muestreados.

\begin{tabular}{|c|c|c|c|c|}
\hline Árbol & Edad & $\begin{array}{c}\text { AT } \\
(\mathrm{m})\end{array}$ & $\begin{array}{c}\text { AC } \\
(\mathrm{m})\end{array}$ & $\begin{array}{c}\text { DAP } \\
(\mathrm{cm})\end{array}$ \\
\hline 1 & 7 & 7,60 & 4,50 & 8,0 \\
\hline 2 & 12 & 9,50 & 6,25 & 13,0 \\
\hline 3 & 13 & 10,5 & 5,60 & 11,5 \\
\hline 4 & 14 & 11,4 & 8,00 & 13,0 \\
\hline 5 & 10 & 7,30 & 3,80 & 8,0 \\
\hline 6 & 12 & 10 & 8,00 & 16,0 \\
\hline 7 & 9 & 7,80 & 5,00 & 10,5 \\
\hline 8 & 15 & 11,4 & 7,40 & 16,0 \\
\hline 9 & 10 & 7,40 & 5,60 & 7,0 \\
\hline 10 & 15 & 11,9 & 9,50 & 15,5 \\
\hline
\end{tabular}

Los árboles fueron apeados y se cortaron rodajas dentro de las dos primeras trozas comerciales. Las alturas de muestreo en el fuste fueron 4 ( 2 en cada troza comercial): $0,3 \mathrm{~m} ; 1,3 \mathrm{~m}$ (altura de pecho=AP); $2,3 \mathrm{~m}$ y $4 \mathrm{~m}$ a partir del suelo. La máxima altura muestreada representa $45-100 \%$ de la altura comercial del fuste.

Sobre cada rodaja se cortó una tabla central sobre la cual se ubicaron los anillos de crecimiento, y se marcaron y obtuvieron las probetas de densidad. Cada probeta contiene 3 anillos de crecimiento desde la médula (posición 0-3 años) a la corteza (posición 12-15 años). Se determinaron la densidad normal al $12 \%$ de contenido de humedad (peso al 12\%/volumen al 12\%). Las probetas se dejaron estacionar durante 2 meses en ambiente controlado hasta alcanzar la humedad de equilibrio higroscópico (12\%). El contenido de humedad se constató como peso en húmedo-peso anhidro/peso anhidro x 100. El volumen se determinó por desplazamiento de fluidos. El peso anhidro se determinó sobre balanza $(0,001 \mathrm{~g})$ luego de secar las probetas en estufa a $103^{\circ} \mathrm{C}$ hasta peso constante.

Sobre esas mismas probetas se cortaron astillas que se colocaron en tubos de ensayo para macerarlos (Franklin 1945) respetando las mismas posiciones radiales que en densidad. Las fibras individuales $(\mathrm{n}=30$ por posición radial y altura de muestreo) se midieron sobre imágenes digitales obtenidas de los macerados con microscopio óptico y cámara digital asociada (Olympus CX31, Japón y Lumenera Infinity, Canadá). La medición se realizó con Image Pro (Media Cibernetics, USA).

Para determinar la edad de transición de madera juvenil a madura, se utilizó el método de regresión segmentada sobre el patrón radial de la propiedad a analizar en cada árbol muestreado. Este método supone que en el patrón radial de la misma (asociado con la edad del anillo de crecimiento) existe un cambio notorio en la pendiente de la línea de regresión de la propiedad en estudio correspondiente a la edad de transición. El modelo de regresión por segmentos general se presenta en la Ecuación 1 (Tasissa and Burkhart 1998).

$$
Y_{i}=\beta_{0}+\beta_{1} x_{i}+\beta_{2}\left(x_{i}-T\right) x_{2}+e_{i}
$$

Donde:

$\mathrm{Y}_{\mathrm{i}}=$ característica de interés en el i-ésimo anillo de crecimiento

$\beta_{0}, \beta_{1}, \beta_{2}=$ coeficientes de regresión

$\mathrm{x}_{\mathrm{i}}=$ edad de formación del i-ésimo anillo de crecimiento

$\mathrm{T}=$ edad de transición de madera juvenil a madura

$\mathrm{x}_{2}=$ variable indicadora : $\mathrm{x}_{2}=0$ si $\mathrm{x}_{\mathrm{i}}<\mathrm{T}$ (madera juvenil) o $\mathrm{x}_{2}=1$ si $\mathrm{x}_{\mathrm{i}}>\mathrm{T}$ (madera madura)

$\mathrm{e}_{\mathrm{i}}=$ error aleatorio asociado al i-ésimo anillo de crecimiento. 
Se aplicó el método Piecewise Linear Regression (Breakpoint Regresión, Statistica v6) para determinar la edad de transición entre madera juvenil y madura en cada altura de muestreo por separado.

Se realizó un ANOVA con posición radial y altura de muestreo como efectos fijos, para testear las diferencias en las distintas alturas de muestreo y en las edades dentro de la misma altura (Ecuación 2). La comparación de medias se testeó con la prueba de Tukey $(\mathrm{p}<0,05)$.

$\mathrm{i}=1,2, \ldots .5 \mathrm{j}=1,2,3,4$

$$
y_{i j}=\mu+\alpha_{i}+\beta_{j}+\varepsilon_{i j}
$$

Donde,

$\mathrm{y}_{\mathrm{ij}}$ : estimación de la variable en la posición radial i, a la altura de muestreo j

$\alpha$ : efecto de la posición radial

$\beta$ : efecto de la altura de muestreo en el fuste

$\varepsilon_{\mathrm{ij}}$ : error experimental

A partir de la edad de transición madera juvenil-madera madura generada para cada altura de muestreo y las diferencias halladas a lo largo del fuste (alturas-ANOVA), se construyó en forma manual un modelo descriptivo de la variación de las propiedades estudiadas en la porción del fuste analizada.

\section{RESULTADOS Y DISCUSION}

\section{Variación axial y radial}

La densidad de la madera incrementó sus valores desde la médula $\left(754 \pm 4 \mathrm{~kg} / \mathrm{m}^{3}\right)$ hacia la corteza $\left(832 \pm 3 \mathrm{~kg} / \mathrm{m}^{3}\right)$ en todas las alturas de muestreo en el fuste (Figura 1). A partir de la probeta de 3-6 años, los valores se mantuvieron radialmente estables sin diferencias significativas entre ellos (Tabla 2, $\mathrm{p}>0,05)$. Axialmente la densidad disminuyó con la altura de muestreo en el fuste pero sin diferencias significativas según el ANOVA (Tabla 2, p>0,05).

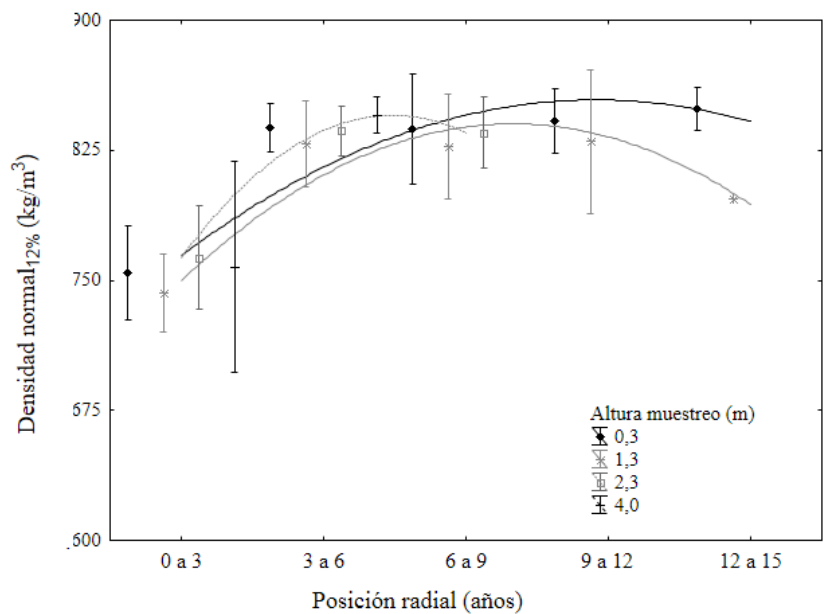

Figura 1. Densidad de la madera según edad y en cuatro alturas en el fuste.

Media \pm DE y línea de ajuste polynomial. 
Tabla 2. Densidad $\left(\mathrm{kg} / \mathrm{m}^{3}\right)$ por posición radial (edad, años) y altura de muestreo $(\mathrm{m})$.

\begin{tabular}{|l|l|l|l|l|l|}
\hline Posición radial & $0-3$ años & $3-6$ años & $6-9$ años & $9-12$ años & $12-15$ años \\
& $754 \mathrm{a}$ & $835 \mathrm{~b}$ & $833 \mathrm{~b}$ & $837 \mathrm{~b}$ & $832 \mathrm{~b}$ \\
& \pm 4 & \pm 2 & \pm 3 & \pm 3 & \pm 3 \\
\hline Posición axial & $0,3 \mathrm{~m}$ & $1,3 \mathrm{~m}$ & $2,3 \mathrm{~m}$ & $4 \mathrm{~m}$ & \\
\hline & $816 \mathrm{a}$ & $801 \mathrm{a}$ & $796 \mathrm{a}$ & $787 \mathrm{a}$ & \\
& \pm 4 & \pm 5 & \pm 4 & \pm 6 & \\
\hline
\end{tabular}

Media \pm desvío estándar. Prueba de Tukey $(\mathrm{p}<0,05)$.

La longitud de fibras también registró un aumento de sus valores desde la médula $(920 \pm 89 \mu \mathrm{m})$ hacia la corteza $(1054 \pm 53 \mu \mathrm{m})$ en todas las alturas de muestreo en el fuste (Figura 2). Radialmente las diferencias significativas se establecieron entre los 0-3 años y el resto, los valores de 3-6 y 6-9 años sin diferencias entre sí y luego se diferencia la posición 9-12 años con la mayor magnitud (Tabla 3). La altura de muestreo no fue una fuente significativas de variación (Tabla 3, p=0,07).

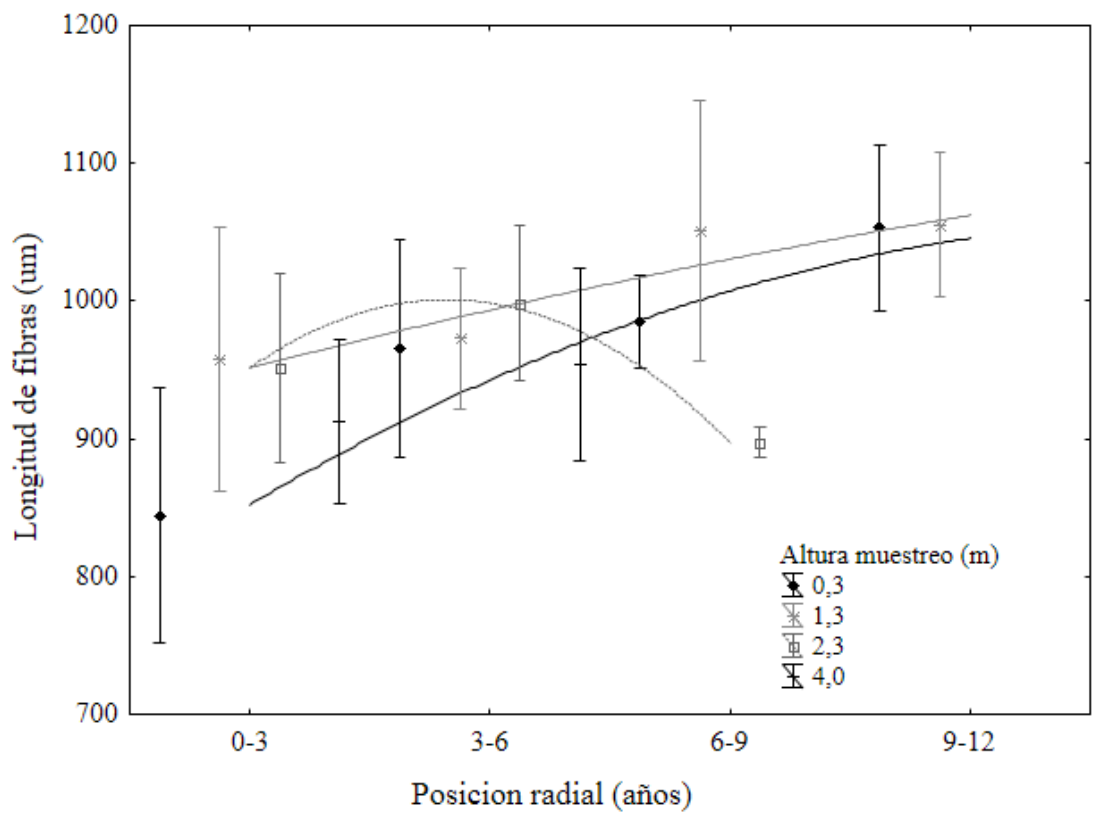

Figura 2. Longitud de fibras según edad y en cuatro alturas en el fuste. Media \pm DE y línea de ajuste polynomial.

Tabla 3. Longitud de fibras $(\mu \mathrm{m})$ por posición radial (edad, años) y altura de muestreo $(\mathrm{m})$.

\begin{tabular}{|l|l|l|l|l|}
\hline Posición radial & $0-3$ años & 3-6 años & $6-9$ años & $9-12$ años \\
\hline & $\begin{array}{l}920 \mathrm{a} \\
\pm 89\end{array}$ & $\begin{array}{l}973 \mathrm{~b} \\
\pm 62\end{array}$ & $\begin{array}{l}998 \mathrm{bc} \\
\pm 80\end{array}$ & $\begin{array}{l}1054 \mathrm{c} \\
\pm 54\end{array}$ \\
\hline Posición axial & $0,3 \mathrm{~m}$ & $1,3 \mathrm{~m}$ & $2,3 \mathrm{~m}$ & $4 \mathrm{~m}$ \\
\hline & $\begin{array}{l}950 \mathrm{a} \\
\pm 103\end{array}$ & $\begin{array}{l}992 \mathrm{a} \\
\pm 84\end{array}$ & $\begin{array}{l}961 \mathrm{a} \\
\pm 67\end{array}$ & $\begin{array}{l}926 \mathrm{a} \\
\pm 63\end{array}$ \\
\hline
\end{tabular}

Media \pm desvío estándar. Prueba de Tukey $(\mathrm{p}<0,05)$. 
Adamopoulus and Voulgaridis (2002) también demostraron el incremento radial de la longitud de fibras en acacia blanca creciendo en Grecia aunque con árboles de edades superiores a los nuestros (1837 años). Estos autores determinaron un incremento de los valores hasta la edad de 7 años. Asimismo, la variación axial que reportaron para las fibras fue no uniforme. Stringer and Olson (1987) publicaron resultados similares con la misma tendencia radial y valores muy similares $(750-1060 \mu \mathrm{m})$ a los de este trabajo en arboles de 10-12 años en Estados Unidos. Sin embargo, la variación axial reportada fue de leve descenso con la altura pero con alturas de muestreo superiores a las de nuestro estudio y que alcanzaron el $80 \%$ de la altura total del árbol.

La ausencia de variación axial de la densidad ya fue reportada para la especie por otros autores (Stringer and Olson 1987, Adamopoulus et al. 2007). Asimismo, los valores medios hallados en densidad $\left(803 \pm 5 \mathrm{~kg} / \mathrm{m}^{3}\right)$ son levemente superiores a los reportados para la especie por otros autores (Forest Products Laboratory 1987, Stringer and Olson 1987, Keil et al. 2011, Pollet et al. 2012). Comparativamente con las maderas utilizadas en el mercado argentino, los valores hallados son mayores a los de los pinos, álamos y Eucalyptus grandis, y similares a los de E. globulus (Atencia 2003). Estos resultados en conjunto (homogeneidad de la densidad en el fuste comercial, comienzo de formación de madera madura a partir de los 3-6 años y altos valores de densidad normal) colocan a esta especie en una situación de ventaja tecnológica frente a otras especies del mercado maderero local.

\section{Edad de transición y Modelos descriptivos}

La edad de transición entre madera juvenil y madera madura calculada por regresión segmentada para cada altura de muestreo se expresa en la Tabla 4 para ambas variables.

Tabla 4. Edad de transición (años) de acuerdo a la densidad básica y la longitud de fibras en acacia blanca.

\begin{tabular}{|l|l|l|l|l|}
\hline Altura muestreo $(\mathrm{m})$ & \multicolumn{2}{|l|}{ Densidad $_{12}\left(\mathrm{~kg} / \mathrm{m}^{3}\right)$} & \multicolumn{3}{c|}{$\begin{array}{c}\text { Longitud de fibra } \\
(\mu \mathrm{m})\end{array}$} \\
\hline & edad & valor & edad & valor \\
\hline 0,3 & $3-6$ & 816 & $3-6$ & 950 \\
\hline 1,3 & $3-6$ & 803 & $6-9$ & 992 \\
\hline 2,3 & $3-6$ & 796 & $3-6$ & 959 \\
\hline 4,0 & $3-6$ & 787 & $3-6$ & 926 \\
\hline
\end{tabular}

Según estos resultados, podría considerarse que a partir de los 3-6 años comienza la formación de madera madura. Para esta especie Adamopoulus and Voulgaridis (2002) calcularon que el cono de madera juvenil se forma hasta los 7-11 años según los patrones radiales de longitud de fibras hallados en árboles de 18-37 años. Mientras que Stringer and Olson (1987) publicaron como edad de transición los 8 años, caracterizando el material por su densidad, extractivos y longitud de fibras.

De acuerdo a los resultados obtenidos de la variación axial y radial de la densidad básica y longitud de fibra, y sumada la edad de transición entre madera juvenil y madura para estas propiedades, se construyeron los modelos de distribución de la madera juvenil y madura en el fuste (Figura 3). 


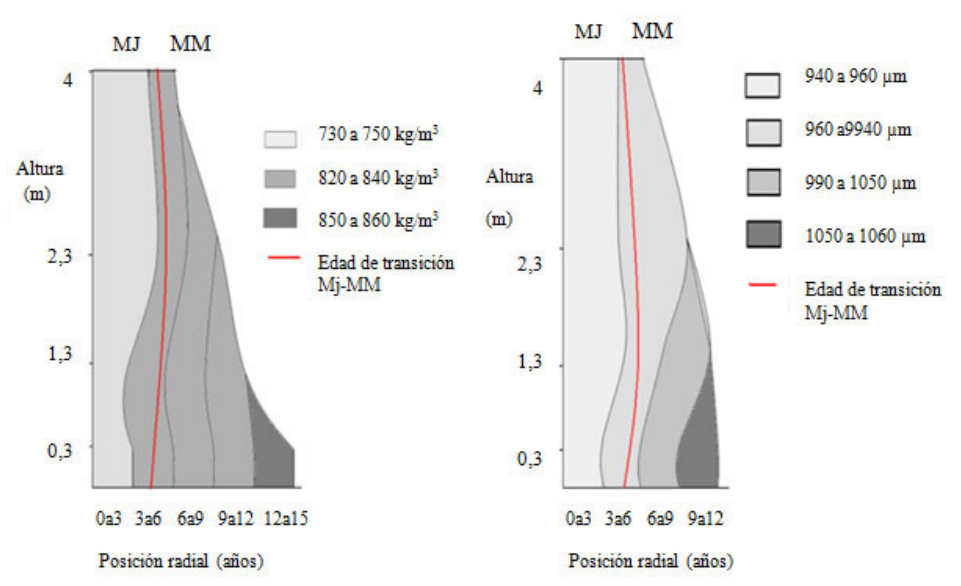

Figura 3. Modelos de distribución de la madera juvenil y madura en el fuste en base a la densidad de la madera y la longitud de fibras en Robinia pseudoacacia.

La madera juvenil recorre todo el fuste muestreado (hasta $4 \mathrm{~m}$ ) como un pequeño cilindro central homogéneo en densidad y longitud de fibras. En la primera troza comercial (hasta los 2,3 m) la madera formada presenta mayor variación en longitud de fibras, mientras que la densidad puede considerarse homogénea. Los valores de madera juvenil fueron de $754 \mathrm{~kg} / \mathrm{m}^{3}$ para densidad y $927 \mu \mathrm{m}$ para longitud de fibras siendo significativamente menores que los de madera madura de $834 \mathrm{~kg} / \mathrm{m}^{3}$ y $994 \mu \mathrm{m}$ respectivamente.

El modelo de madera juvenil y madura hallado en este trabajo (Figura 3) y con el diseño de muestreo aplicado, se asemeja al modelo de cilindro central reportado para coníferas. Este modelo fue citado por Zobel and Sprague (1998) para caracterizar el modelo más difundido en latifoliadas, considerando un cilindro juvenil pero de menor diámetro en el ápice.

\section{CONCLUSIONES}

La densidad de la madera de los árboles estudiados de Robinia pseudoacacia con edades comprendidas entre 7-15 años, mostró una escasa variación a lo largo del fuste comercial (sin variación axial y escasa radial), por lo tanto puede considerarse un materia prima tecnológicamente uniforme en densidad. El rango de magnitud en la densidad normal la clasifica como madera semi-pesada $\left(750\right.$ y $\left.823 \mathrm{~kg} / \mathrm{m}^{3}\right)$. La longitud de fibras mostró aumento radial de sus valores de médula a corteza y homogeneidad axial. Los rangos de valores hallados $(963 \mu \mathrm{m} \pm 38)$ para esta importante propiedad fueron acordes a los hallados en la literatura para la especie.

El modelo de madera juvenil y madura hallado en este trabajo y con el diseño de muestreo aplicado, se asemeja al modelo de cilindro central. El cono juvenil es pequeño y se formaría hasta la edad de 3-6 años. 


\section{AGRADECIMIENTOS}

Los autores quieren agradecer la colaboración del Ing. Hernán Belossi de la Empresa Andreoli S.A, quien realizó el muestreo de los árboles. Al Ing. Miguel Tortoriello y a la Ing. María José Castillo del Laboratorio de Ensayos de Materiales y Estructuras (LEMEJ), de la Universidad Nacional del Noroeste donde se realizaron los ensayos.

\section{REFERENCIAS}

Adamopoulos, S.; Voulgaridis, E. 2002. Within-tree variation in growth rate and cell dimensions in the wood of black locust (Robinia pseudoacacia). IAWA J 23 (2): 191-199.

Adamopoulos, S.; Passialis, C.; Voulgaridis, E. 2007. Strength properties of Juvenile and Mature wood in Black locust (Robinia pseudoacacia L.). Wood and Fiber Science 39 (2): 241-249.

Adamopoulos, S.; Passialis, C.; Voulgaridis, E. 2010. Ring width, latewood proportion and density relationships in black locust wood of different origins and clones. IAWA Journal 31 (2): 169178.

Atencia, M.E. 2003. Densidad de maderas $\left(\mathrm{kg} / \mathrm{m}^{3}\right)$ ordenadas por nombre común. INTI, CITEMA, Argentina. 8 p.

Barnett, J.; Jeronimidis, G. 2003. Wood Quality and its biological basis. CRC Press and Blackwell Publ. Ltd., U.K

Bhat, K.M.; Priya, P.B.; Rugmini, P. 2001. Characterization of juvenile wood in teak. Wood Science and Technology 34:517-532.

Cobas, A.C.; Area, M.C.; Monteoliva, S. 2013a. Transición de madera juvenil a madura en Populus deltoides implantado en Buenos Aires, Argentina. Maderas. Ciencia y Tecnología 15 (2): 223234.

Cobas, A. C.; Felissia, F.; Monteoliva, S.; Area, M.C. 2013b. Optimization of the properties of poplar and willow chemimechanical pulps by a mixtures design of juvenile and mature wood. BioResources 8 (2):1646-1656.

Cobas, A. C.; Area, M.C.; Monteoliva, S. 2014. Patrones de variación de la densidad de la madera y morfometría celular de Salix babylonica var sacramenta para la determinación de la edad de transición entre madera juvenil y madura. Maderas. Ciencia y Tecnología 16 (3):343-354.

De Bell, D.S.; Singleton, R.; Harrington, C.A.; Gartner, B.L. 2002. Wood density and fiber length in young Populus stem: relation to clone, age, growth rate, and pruning. Wood and Fiber Science 34 (4):529-539.

Downes, G.; Hudson, I.; Raymond, C.; Dean, A.; Micheli, A.; Schimlek, L.; Evans, R. Muneri, A. 1997. Sampling plantations Eucalyptus for wood and fiber properties. CSIRO Publishing, Australia.

Downes, G.M.; Drew, D.; Bataglia, M.; Schulze, D. 2009. Measuring and modelling stem growth and wood formation: An overview. Dendrochronologia 27:147-157. 
Forest Products Laboratory. 1987. Wood Handbook: Wood as an engineering material. U.S. Dep. Agric. 72 p.

Franklin, G.L. 1945. Preparation of thin sections of synthetic resins and wood-resin composites, and a new macerating method for wood. Nature 155 (3924):51.

Fukasawa, K. 1984. Juvenile wood of hardwoods judged by density variation. IAWA Bulletin n.s. $5(1): 65-73$.

Gartner, B.L.; Lei, H.; Milote, M.R. 1997. Variation in the anatomy and specific gravity of wood between and within trees of red alder (Alnus rubra). Wood and Fiber Science 29 (1):10-20.

Keil, G.; Spavento, E.; Murace, M.; Millanes, A. 2011. Acacia blanca (Robinia pseudoacacia L.) y Acacia negra (Gleditsia triacanthos L.): aspectos tecnológicos relacionados al empleo en productos de madera maciza. Forest Systems 20 (1):21-26.

Kojima, M.; Yamamoto, H.; Yoshida, M.; Ojio, Y.; Okumura, K. 2009a. Maturation property of fast-growing hardwood plantation species: A view of fiber length. Forest Ecology and Management $257: 15-22$.

Kojima, M.; Yamamoto, H.; Marsoem, S.N.; Okuyama,T.; Yoshida, M.; Nakai,T.; Yamashita, S.; Saegusa, K.; Matsune, K.; Nakamura, K.; Inoue, Y.; Arizono, T. 2009b. Effects of the lateral growth rate on wood quality of Gmelina arbórea from 3.5-, 7- and 12-year-old plantations. Ann For Sci 66: 507 .

Larson, P.R.; Kretschmann, D.E.; Clark III, A.; Isebrands, J.G. 2001. Formation and properties of juvenile wood in southern pines: a synopsis. General Technical Report FPL-GTR 129, Madison, WI. U.S. Department of Agriculture, Forest Service, Forest Products Laboratory, 42p.

Matyas, C.; Peszlen, I. 1997. Effect of age on selected wood quality traits of poplar clones. Silvae Genetica 46 (2-3):64-72.

Moya Roque, R.; Berrocal, A.; Serrano, J.R.; Tomazello Filo, M. 2009. Variación radial de la anatomía, densidad y durabilidad de la madera de teca (Tectona grandis) procedente de dos calidades de sitio y dos regiones climáticas de Costa Rica. Investigación Agraria: Sistemas y Recursos Forestales 18(2): 119-131.

Moya Roque, R.; Tomazello Filo; M. 2009. Variação radial da estrutura anatômica do lenho de árvores de Gmelina arborea em diferentes condições de clima e de manejo na Costa Rica. Sci For 37 (83):273-285.

Panshin, A.; De Zeeuw, C. 1980. Textbook of wood Technology. McGraw-Hill Book Company.

Pollet, C.; Verheyen, C; Hébert, J.; Jourez, B. 2012. Physical and mechanical properties of black locust (Robinia pseudoacacia) wood grown in Belgium. Can J For Res 42:831-840.

Stringer, J.W.; Olson, J.R. 1987. Radial and vertical variation in stem properties of juvenile black locust (Robinia pseudoacacia). Wood and Fiber Science 19 (1):59-67.

Tasissa, G.; Burkhart, H.E. 1998. Juvenile-mature wood demarcation in loblolly pine trees. Wood and Fiber Science 30 (2):118-127.

Tsuchiya, R.; Furukawa, I. 2009. Radial variation in the size of axial elements in relation to stem increment in Quercus serrata. IAWA J 30 (1):15-26.

Zobel, B.J.; Jett, J.B. 1995. Genetics of wood production. Springer-Verlag, Berlín. 
Zobel, B.J.; Sprague, J.R. 1998. Juvenile wood in forest trees. Springer-Verlag, Berlín. 